\title{
Exploiting the radio signal from air showers: the AERA progress
}

\author{
B. Revenu ${ }^{1, a}$ for the Pierre Auger Collaboration ${ }^{2}$ \\ ${ }^{1}$ Subatech, Université de Nantes, École des Mines de Nantes, CNRS/IN2P3, Nantes, France \\ ${ }^{2}$ For the complete author list, see http://www.auger.org/archive/authors_2016_09.html
}

\begin{abstract}
We present the lastest results and status of the Auger Engineering Radio Array (AERA), located within the Pierre Auger Observatory. AERA, with more than 150 radio stations spread over $17 \mathrm{~km}^{2}$, is the largest radio detector in the world for extensive air showers above $10^{17} \mathrm{eV}$. The electric field emitted by secondary electrons and positrons allows us to estimate all characteristics of the primary cosmic ray: arrival direction, energy and mass composition. The performance of AERA together with the analysis methods are described. The final aim of AERA is mainly to improve the composition estimation of ultra-high energy cosmic rays as a standalone detector or in association with other instruments such as a ground particle detector or a fluorescence telescope.
\end{abstract}

\section{Introduction}

There are many aspects still not fully understood in the field of ultra-high energy cosmic rays. For the past decade, a clear energy cutoff has been observed in the energy spectrum around $4 \times 10^{19} \mathrm{eV}$ (see [1$3]$ ). Nevertheless, the sources are still unidentified as no significant clustering has been discovered at the highest energies and no departure from isotropic expectations are observed at lower energies at any angular scales [4]. Determining the composition could be the missing key to a much better understanding of the origin of ultra-high energy cosmic rays. Air showers are produced after a first collision of the primary cosmic ray with the atmosphere's constituent nuclei. The secondary electrons and positrons of these showers emit a coherent radio signal in the range 1-300 MHz. This signal allows us to reconstruct the arrival direction and the energy of the cosmic ray, together with the shower's depth of maximum development $X_{\max }$ which is essential to estimate its nature [5-7]. The uncertainty on the $X_{\max }$ is of the same order as that achieved using the fluorescence light $\left(\sim 20 \mathrm{~g} / \mathrm{cm}^{2}\right.$ [8]) emitted by the atmospheric nitrogen excited by the air shower. A strong asset of the radio technique is its duty cycle of around $100 \%$ compared to $14 \%$ with a fluorescence telescope [9].

\section{The Auger Engineering Radio Array}

The Auger Engineering Radio Array is the largest radio detector of cosmic rays in the world. It is located at the Pierre Auger Observatory [10] and covers an area of $17 \mathrm{~km}^{2}$. The Observatory contains a surface detector (SD) of 1660 water Cherenkov tanks spread over $3000 \mathrm{~km}^{2}$, a fluorescence detector (FD) of 27 telescopes at 4 sites around the SD, and the AMIGA instrument with burried

\footnotetext{
a e-mail: revenu@in2p3.fr
} 
scintillators that allows the measurement of the muonic component of air showers. As of the end of 2016, AERA consists of 153 autonomous radio stations running continuously thanks to solar panels and batteries. Radio stations with log-periodic dipole antenna (LPDA) composed the first AERA setup of 24 detectors in 2011, covering $0.6 \mathrm{~km}^{2}$ and communicating through fibers with the central data acquisition system. In 2013, 100 new stations with butterfly antenna were installed and 29 more in 2015 to complete the actual setup which is presented in FIG 1. The electric field is recorded in the band $30-80 \mathrm{MHz}$ with a sampling rate of $180 \mathrm{MHz}$ or $200 \mathrm{MHz}$ depending on the electronics in use. The detectors run in self-trigger mode together with an external trigger coming from the SD and the

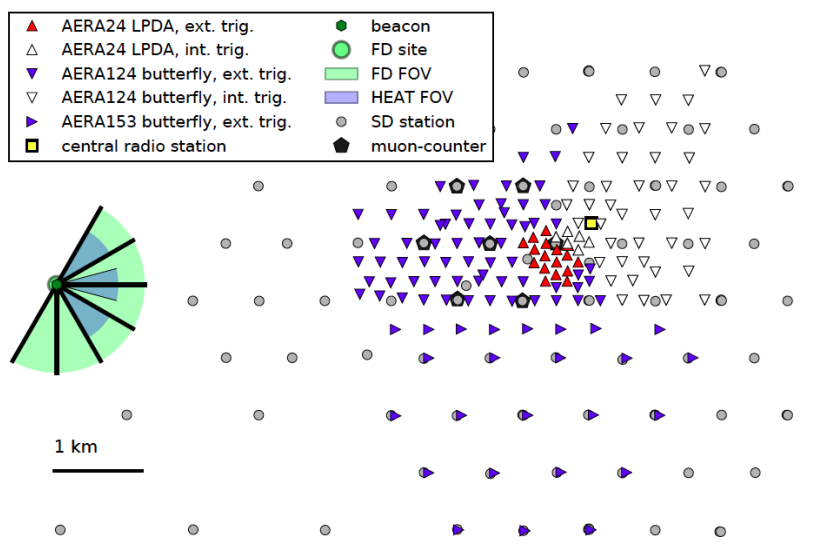

Figure 1. The AERA antennas (triangles) among the other instruments of the Pierre Auger Observatory (water Cherenkov tanks of the SD, fluorescence telescopes and AMIGA muon counters).

FD. The results presented here are those obtained using the external trigger mode.

\section{Calibration}

The radio stations are calibrated in both time and amplitude. For timing (absolute and relative), we use GPS receivers in conjunction with a reference beacon located at the closest FD site (see FIG 1) and signals emitted by commercial airplanes passing over the AERA site. We obtain an accuracy better than $2 \mathrm{~ns}$ [11]. For the amplitude, we first used weather balloon flights equipped with a calibrated transmitter. For cost reasons, we preferred to use a drone carrying the same kind of calibrated light source. The overall accuracy, taking into account all systematic errors, is 9.3\% [12], on a LPD antenna. We plan to repeat this calibration procedure to the butterfly antennas.

\section{The radio signal from air showers}

The electric field emitted by air showers is the superposition of two distincts mechanisms. In the frequency range of interest in AERA (30-80 MHz), the dominant contribution is the geomagnetic effect: the systematic opposite drift of positrons and electrons during their propagation in the geomagnetic field results in an average transverse current leading to an electric field polarized in the direction of $\mathbf{v} \times \mathbf{B}$, where $\mathbf{v}$ is the direction of the shower axis and $\mathbf{B}$ is the geomagnetic field direction. The electric field direction does not depend on the observer's location. 
In addtion to the geomagnetic mechanism, the excess of electrons over positrons in air showers leads to another source of electric field, known as the charge-excess contribution. This net negative charge is due to the fast in-flight annihilation of positrons and to the extraction of electrons from the medium. The electric field produced through this mechanism is radially polarized (with respect to the shower axis) and therefore, depends on the observer's location.

A given observer will measure the superposition of both mechanisms as shown in FIG 2. This means that both the amplitude and polarization depend on the ground coordinate with respect to the shower axis. Both mechanisms have been clearly seen in the data thanks to the significant progress of
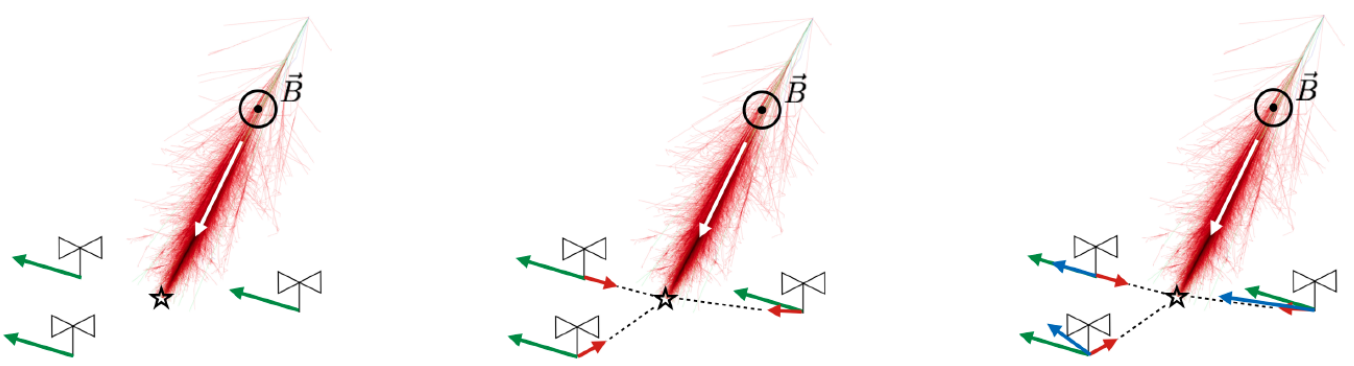

Figure 2. Scheme of an air shower falling between three observers, symbolized by the butterfly antennas. The geomagnetic field $\mathbf{B}$ is indicated. The electric fields produced by the geomagnetic and charge-excess mechanisms are indicated in green and red respectively. The observer measures the superposition of both electric fields, in blue.

the simulation codes these past few years [13-15]. The impressive agreement between the data and the simulation allows us to use the simulation to extract all characteristics of the primary cosmic ray.

\section{Extracting the primary cosmic ray characteristics}

As argued in the previous section, the amplitude and polarization of the electric field depend on the observer's location. We cannot describe the electric field using a simple 1D lateral distribution function (LDF). A 2D LDF has been proposed [16], using the code CoREAS [17], as a model of ground distribution of the electric field. This LDF permits us to estimate the amount of energy emitted as radiation in $30-80 \mathrm{MHz}$ from the cosmic ray. We first correct the data recorded by each station participating in an event from the antenna and electronics responses. After this step, we obtain the electric field as a function of time; we can afterwards compute the energy fluence (energy per unit area) using the time integration of the associated Poynting vector. Then we fit the 2D LDF to extract the ground distribution of the energy fluence that we can integrate over space to get the total energy released as radiation. This radiated energy is rescaled by a factor $1 / \sin ^{2} \alpha$ to correct for the angular separation $\alpha$ from the geomagnetic field. The final estimator is strongly correlated (quadratic scaling as expected for coherent radiation) with the energy of the primary cosmic ray and we obtain an energy of $15.8 \pm 0.7$ (stat) \pm 6.7 (sys) $\mathrm{MeV}$ for a primary energy of $10^{18} \mathrm{eV}$ and an angular separation from the geomagnetic field of $90^{\circ}[18,19]$.

Concerning the reconstruction of the shower $X_{\max }$, the method is also based on simulations. There are several methods in use in AERA, but as an illustration we can compare the measured amplitudes for an actual event to simulated amplitudes using a simulation code (here SELFAS [20]). We use the fact that showers initiated by light or heavy nuclei have very different electric field distributions 
at ground level. Very roughly, young showers (initiated by light nuclei) have strong and peaked distributions when old showers (initiated by heavy nuclei) have weaker and wider distributions. Using a set of simulated showers initiated by protons and iron nuclei with the same arrival direction as an actual shower, we obtain the best agreement of the 2D profiles between the data and the simulation for showers in a preferred range of $X_{\max }$. This way, we have an estimation of the best $X_{\max }$ together with the confidence interval [7], which in turn gives important constraints on the nature of the primary cosmic ray. Working on a set of air showers detected in coincidence by both the FD and AERA, we can compare in FIG 3 the $X_{\max }$ estimated using the radio signal with the FD direct measurement. The correlation is very clear and proves that the radio signal from air showers allows the measurement of the $X_{\max }$.

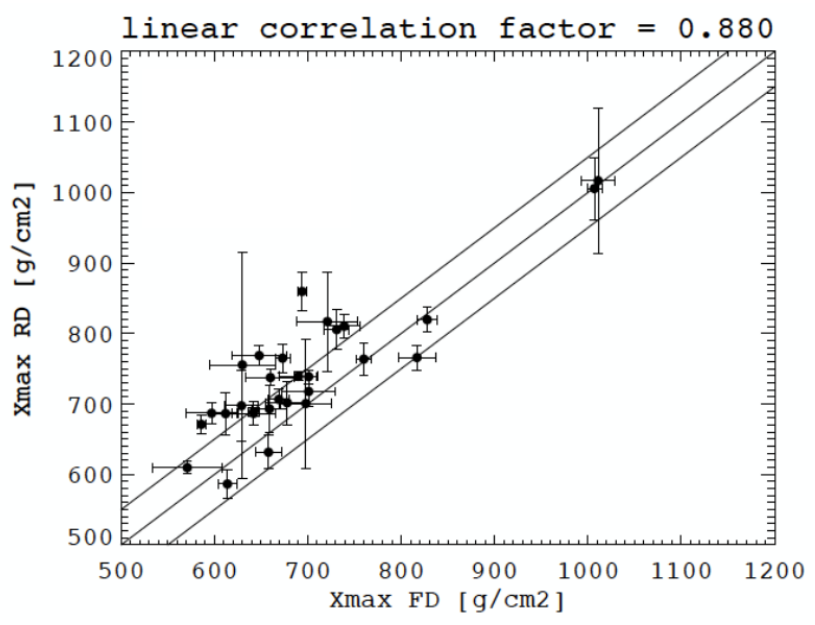

Figure 3. Correlation between the $X_{\max }$ estimated using the radio signal and the $X_{\max }$ measured by the fluorescence light for showers detected in coincidence by AERA and the Pierre Auger Observatory fluorescence detector.

\section{Conclusion}

AERA has been running continuously since 2011 and detects air showers also measured by the other instruments of the Pierre Auger Observatory providing an ideal observation of showers with 4 different observables. AERA contains 153 radio stations spread over $17 \mathrm{~km}^{2}$. The radio signal contains the information needed to reconstruct all characteristics of the primary cosmic rays, in particular their energy and the $X_{\max }$ of the showers they initiate when entering our atmosphere. One of the next steps will be to provide the composition of ultra-high energy cosmic rays using the radio signal.

\section{References}

[1] J. Abraham et al. (Pierre Auger Collaboration), Phys.Lett. B685, 239 (2010), 1002 . 1975

[2] R.U. Abbasi et al. (HiRes), Phys. Rev. Lett. 100, 101101 (2008), astro-ph/0703099

[3] T. Abu-Zayyad et al. (Telescope Array), Astrophys. J. 768, L1 (2013), 1205.5067

[4] P.L. Ghia (Pierre Auger), PoS ICRC2015, 034 (2016) 
[5] P.A. Bezyazeekov et al. (Tunka-Rex), JCAP 1601, 052 (2016), 1509. 05652

[6] S. Buitink, A. Corstanje, H. Falcke, J.R. Hörandel, T. Huege, A. Nelles, J.P. Rachen, L. Rossetto, P. Schellart, O. Scholten et al., Nature 531, 70 (2016), 1603.01594

[7] F. Gaté, The Pierre Auger Collaboration, $X_{\text {max }}$ reconstruction from amplitude information with AERA, in Proceedings of the ARENA 2016 workshop (Groningen, The Netherlands) (ARENA, 2016)

[8] J. Abraham et al. (Pierre Auger Collaboration), Phys.Rev.Lett. 104, 091101 (2010), 1002 .0699

[9] J. Abraham et al. (Pierre Auger Collaboration), Nucl.Instrum.Meth. A620, 227 (2010), 0907. 4282

[10] A. Aab et al. (Pierre Auger), Nucl. Instrum. Meth. A798, 172 (2015), 1502.01323

[11] A. Aab et al. (Pierre Auger), JINST 11 (2016), 1512 . 02216

[12] R. Krause, The Pierre Auger Collaboration, Detection of High-Energy Cosmic Rays with the Auger Engineering Radio Array, in Proceedings of the 14th VCI, Vienna (Elsevier, 2014)

[13] V. Marin, the CODALEMA Collaboration, Charge excess signature in the CODALEMA data. Interpretation with SELFAS2, in proceedings of the 32nd ICRC, Beijing, China (ICRC, 2011)

[14] The Pierre Auger Collaboration, Phys. Rev. D 89 (2014)

[15] A. Belletoile, R. Dallier, A. Lecacheux, V. Marin, L. Martin, B. Revenu, D. Torres Machado, Astroparticle Physics 69, 50 (2015)

[16] A. Nelles et al., JCAP 1505, 018 (2015), 1411.7868

[17] T. Huege, M. Ludwig, C. James, Simulating radio emission from air showers with CoREAS, in Proceedings of the ARENA 2012 workshop (Erlangen, Germany) (to be published), AIP Conference Proceedings

[18] A. Aab et al. (Pierre Auger), Phys. Rev. D93, 122005 (2016), 1508.04267

[19] A. Aab et al. (Pierre Auger), Phys. Rev. Lett. 116, 241101 (2016), 1605.02564

[20] V. Marin, B. Revenu, Astropart.Phys. 35, 733 (2012), 1203. 5248 